Check for updates

Cite this: RSC Adv., 2018, 8, 28395

\title{
Inkjet printing and UV-LED curing of photochromic dyes for functional and smart textile applications $\uparrow$
}

\author{
Sina Seipel, (D) *a Junchun Yu, (D) a Aravin P. Periyasamy, (D) ${ }^{\mathrm{b}}$ Martina Viková, (D) b \\ Michal Vik (D) ${ }^{b}$ and Vincent A. Nierstrasz (D) ${ }^{a}$
}

Health concerns as a result of harmful UV-rays drive the development of UV-sensors of different kinds. In this research, a UV-responsive smart textile is produced by inkjet printing and UV-LED curing of a specifically designed photochromic ink on PET fabric. This paper focuses on tuning and characterizing the colour performance of a photochromic dye embedded in a UV-curable ink resin. The influence of industrial fabrication parameters on the crosslinking density of the UV-resin and hence on the colour kinetics is investigated. A lower crosslinking density of the UV-resin increases the kinetic switching speed of the photochromic dye molecules upon isomerization. By introducing an extended kinetic model, which defines rate constants $k_{\text {colouration, }} k_{\text {decay }}$ and $k_{\text {decolouration, }}$ the colour performance of photochromic textiles can be predicted. Fabrication parameters present a flexible and fast alternative to polymer conjugation to control kinetics of photochromic dyes in a resin. In particular, industrial fabrication parameters during printing and curing of the photochromic ink are used to set the colour yield, colouration/decolouration rates and the durability, which are important characteristics towards the development of a UV-sensor for smart textile applications.

Received 9th July 2018

Accepted 28th July 2018

DOI: $10.1039 / \mathrm{c} 8 \mathrm{ra0} 5856 \mathrm{c}$

rsc.li/rsc-advances molecular structure of the dye, which is reversible either through visible light or heat. The isomers A and B of the dye have different absorption spectra. ${ }^{7}$

In recent literature, the development of UV-sensing textiles using photochromic dyes has been paid much attention. The integration of photochromic dyes into textile structures has been proven successful with traditional textile production techniques such as screen-printing, ${ }^{8-10}$ dyeing ${ }^{11,12}$ and mass dyeing. ${ }^{10,13,14}$ Moreover, the application of photochromic dyes using novel production techniques has emerged. Whereas, Aldib $^{15}$ investigated the potential of digital inkjet printing of solvent-based ink to produce photochromic textiles, Fu et al. ${ }^{16}$ studied photonic curing to produce a photochromic crosslinked polymer. In other areas of textile colouration such as pigment printing, the advantages of inkjet printing in combination with photo-curing of an aqueous polyurethane acrylate system ${ }^{17}$ or by mini-emulsion encapsulation ${ }^{18}$ were explored. To the best of our knowledge, the combination of digital inkjet printing and UV-LED curing has not yet been studied in the industrially applicable fabrication of a UV-sensing smart textile using photochromic dye.

UV-curable coatings distinguish themselves from thermally dried coating or binder systems, such as water-based acrylate binders typically used in screen-printing, by their high rigidity and three-dimensional cross-linked structure. ${ }^{19}$ According to Schwalm et al. ${ }^{20}$ and Agarwal, ${ }^{21}$ depending on the chemistry, i.e. the choice of oligomers and monomers in the formulation, the coating's flexibility, adhesion and hardness can be controlled.
${ }^{a}$ Textile Materials Technology, Department of Textile Technology, Faculty of Textiles, Engineering and Business, University of Borås, 50190 Borås, Sweden. E-mail: sina. seipel@hb.se

${ }^{b}$ Department of Material Engineering, Faculty of Textile Engineering, Technical University of Liberec, 46117 Liberec, Czech Republic

$\dagger$ Electronic supplementary information (ESI) available. See DOI: $10.1039 / \mathrm{c} 8 \mathrm{ra05856c}$ 
Substrate-ink adhesion and flexibility are particularly important when printing on flexible substrates like textiles. In general, UVcurable inkjet inks exhibit good adhesion to porous substrates as compared to solvent-based alternatives. ${ }^{22}$ Inkjet printing and UV-curing also provide a controllable process, where the thickness of the printed ink and the cross-linked network can be controlled. Eventually, a high degree of crosslinking is desired to achieve good adhesion of dyes in a solid ink matrix onto the textile surface and to ensure a durable functional surface treatment. The degree of polymer crosslinking of the carrying ink also affects the rate of the switching reaction of photochromic dyes such as spirooxazines or naphthopyrans between their ring-closed colourless and ring-opened coloured state (Fig. 1(a)). A $90^{\circ}$ rotation in the molecular structure between non-planar and planar isomers requires space., ${ }^{7,23,24}$ Hence, the structural molecular changes of the dyes upon isomerization are largely affected by the properties of the host matrix. Whereas, Ercole et al. ${ }^{25}$ and Malic et al. ${ }^{26}$ studied the placement of naphthopyran dye in host polymers, Mutoh et al. ${ }^{27}$ investigated block copolymers as host matrix. Other factors, which influence the photochromic response are chain length, free volume and rigidity of the host matrix..$^{2427,28}$ In particular, the higher the crosslinking density of the ink, the lower the degree of freedom in the matrix and hence the slower the switching reaction of the photochromic dye is. ${ }^{25,26,29}$

In this paper, we explore and tune the colour performance of a novel UV-responsive textile using fabrication parameters of an industrially applicable process. Altering the curing intensity of the photochromic ink on the textile during fabrication, influences the rigidity of the UV-resin, the dye-polymer matrix interaction and hence the degree of freedom of the dye to switch between isomers in the matrix. In particular, the photo-switching of the

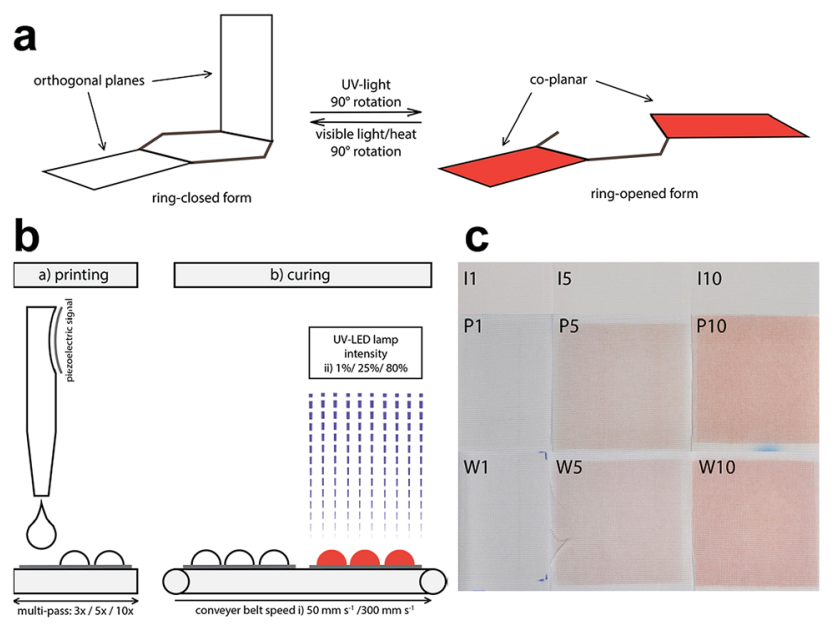

Fig. 1 (a) Isomerization of a photochromic dye between its colourless ring-closed and coloured ring-opened form. The planes indicate the remaining chemical groups of the heterocyclic organic compound that partially undergo a $90^{\circ}$ rotation followed by cleavage of the spiro-carbonoxygen. (b) Production process scheme of photochromic prints. (1) Printing of various passes of the UV-curable photochromic ink on PET. (2) Substrate transportation with a belt speed of $50 \mathrm{~mm} \mathrm{~s}^{-1}$ or $300 \mathrm{~mm} \mathrm{~s}^{-1}$ and curing with 1,25 or $80 \%$ of the maximum UV-LED lamp power. (c) Inactive (I) and active printed (P) and washed (W) photochromic prints with 1,5 and 10 printing passes cured at $50 \mathrm{~mm} \mathrm{~s}^{-1}$ and $80 \%$. photochromic textiles is influenced by the combination of two challenging functions of electromagnetic radiation during production and characterization. The photochromic textile, which is fabricated by inkjet printing and UV-LED curing is activated by UV-light once in use. The impact of UV-rays during curing and activation requires the introduction of an extended kinetic model to describe the kinetics of UV-curable photochromic materials. The lowest curing intensity achieves highest colour yields and fastest switching behaviour between the coloured and colourless state of the photochromic dye Ruby Red in a UV-curable resin. The proposed kinetic model is an important step towards the development of a smart textile UV-sensor and allows the prediction of the colour performance based on fabrication parameters.

\section{Materials and methods}

\section{Materials}

A commercial heterocyclic spiro-compound (Reversacol Ruby Red) from Vivimed Labs, UK was used as photochromic dye in a UVcurable inkjet ink formulation to produce a textile UV-sensor. The photochromic ink contains a dye concentration of $2.5 \mathrm{~g} \mathrm{~L}^{-1}$. The UV-curable carrier consists of dipropylene glycole diacrylate monomers (DPGDA), amine modified polyetheracrylate oligomers (Ebecryl 81) supplied by Allnex SA/NV, Belgium and a UV-LED photo-initiator (Genocure TPO-L) supplied by Rahn AG, Switzerland. Ethyl acetate, 99.9\% (Chromasolv Plus), purchased from Sigma-Aldrich was used as media to disperse the photochromic dye in the ink formulation, but was removed afterwards by vacuum pumping. Plain-woven polyester (PET) fabric with a weight of $147 \mathrm{~g}$ $\mathrm{m}^{-2}$ and 20 and 23 threads $\mathrm{cm}^{-1}$ in warp and weft direction, respectively, received from FOV Fabrics, Sweden was used as the substrate for digital inkjet printing.

\section{Production of photochromic prints}

Photochromic prints were produced using a Sapphire QS 10 print head from Fujifilm Dimatix, USA. Printing was carried out in multi-pass mode with a resolution of $300 \mathrm{dpi}$ and at a print head temperature of $35{ }^{\circ} \mathrm{C}$. To determine the effect of the amount of ink deposition on the crosslinking density and colour behaviour, different amounts of ink were deposited during printing. 1, 3, 5, 7 and 10 passes of ink were printed on PET, which correspond to ink amounts of 3, 8, 10, 13 and $19 \mathrm{~g}$ $\mathrm{m}^{-2}$, respectively. Subsequently, the prints were cured using a UV-LED lamp FireJet from Phoseon Technology, USA with emission wavelengths of 380 to $420 \mathrm{~nm}$ and a maximum emission power of $6 \mathrm{~W} \mathrm{~cm} \mathrm{~cm}^{-2}$ (the UV-LED lamp is part of a continuous pilot-scale inkjet printing system with a conveyer belt for substrate transportation). To determine the optimal curing efficiency and colour performance, curing conditions were varied. Hence, two different conveyer belt speeds, $50 \mathrm{~mm}$ $\mathrm{s}^{-1}$ and $300 \mathrm{~mm} \mathrm{~s}^{-1}$, and three different lamp intensities, $1 \%$, $25 \%$ and $80 \%$, were used (Fig. 1(b)). To determine the effect of a cleaning step, one set of samples was washed once after printing and curing. Washing was done using procedure $4 \mathrm{~N}$ and reference detergent 3 according to ISO 6330:2012. The samples were dried using drip flat drying. 


\section{UV-Vis spectroscopy}

UV-Vis spectra were collected using a Libra S60 double beam spectrophotometer from Biochrom, UK and a UV-3101PC from Shimadzu, Japan double beam spectrophotometer with integrated sphere accessory to affirm fit of the photo-initiator for the UV-LED lamp of the pilot-scale inkjet printing system and to determine the influence of UV-varnish on the absorption spectra of the dye.

\section{Colour analysis}

The colour behaviour of the photochromic prints was measured using an LCAM Photochrom 3 spectrophotometer, which allows continuous colour measurement upon cycles of UV-exposure and relaxation ${ }^{30}$ (Fig. 2).

The activation light source was an Edixon UV-LED lamp (EDEV-3LA1) with a radiometric power $\Phi_{\mathrm{V}}$ of $350 \mathrm{~mW}$ and an emission peak with wavelengths between $395 \mathrm{~nm}$ and $410 \mathrm{~nm}$. The measuring light source was a dual light source system of combined high power white LEDs with CCTs of 4000, 5000 and $7000 \mathrm{~K}$. The measuring light source system illuminates samples with illuminance of $60 \mathrm{klx}$. This illuminance is a compromise between brightest sunlight intensity (120 klx) and daylight intensity without direct sunlight at noon $(20 \mathrm{klx})$. In addition, the measuring light source is equipped with a set of high-pass filters preserving light transmission bellow $420 \mathrm{~nm}$, which decrease possible activation of photochromic composition. Colour values $K / S$ were measured from reflectance values $R$ using the Kubelka-Munk function:

$$
\frac{K}{S}=\frac{(1-R)^{2}}{2 R}
$$

The kinetic model shown in eqn (2) follows first-order kinetics and is generally used to describe photochromic colour behaviour for both the colouration and decolouration reaction as seen in Fig. $2 .^{31}$

$$
\Delta\left(\frac{K}{S}\right)=\left[\left(\frac{K}{S}\right)_{0}-\left(\frac{K}{S}\right)_{\infty}\right] \mathrm{e}^{-k t}+\left(\frac{K}{S}\right)_{\infty}
$$

Based on the kinetic model, the sensor functionality was specified by its achieved colour intensity $\Delta K / S$ upon activation with UV-light, its rate constant of colour increase $k_{\text {colouration }}$ to achieve

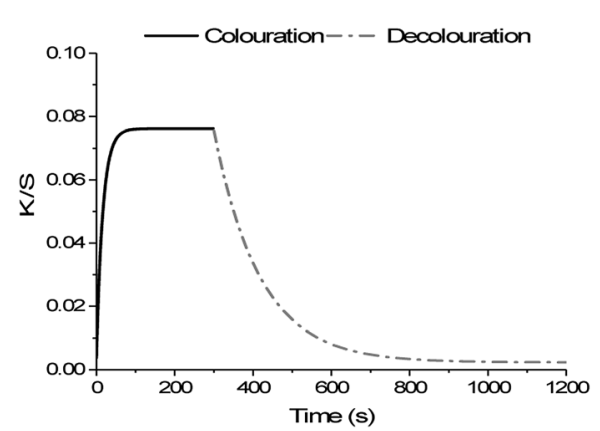

Fig. 2 Scheme of colour measurement cycle with $300 \mathrm{~s}$ of UVexposure for colouration and $900 \mathrm{~s}$ of relaxation for decolouration. maximum colouration $K / S_{\infty}$, and its rate constant of colour decrease $k_{\text {decolouration }}$ to revert to the initial colourless state $K / S_{0}$.

\section{DSC analysis}

The influence of deposited ink amount and curing conditions on the curing efficiency was determined indirectly by analysing the shift of the melting peak $T_{\mathrm{m}}$ of PET using a Q1000 DSC from TA Instruments, USA. The DSC samples sealed in aluminum pans were weighted. The balance (Kern) has $99.96 \%$ accuracy when calibrated against a standard weight of $100 \mathrm{mg}$. The heat flow of samples of about $5.0 \mathrm{mg}$ was measured during a heating and cooling cycle from $25{ }^{\circ} \mathrm{C}$ to $290{ }^{\circ} \mathrm{C}$ and back to $25{ }^{\circ} \mathrm{C}$ with a rate of $10{ }^{\circ} \mathrm{C} \min ^{-1}$. The released energy $\Delta H$ of the endothermic reaction during melting of the material gives indirect information on the degree of polymer crosslinking at the interface between the PET fabric and the photochromic UVcured ink. The shift of the melting peak $T_{\mathrm{m}}$ towards higher temperatures compared to the $T_{\mathrm{m}}$ of pure PET fabric indicates that an insulating layer is built surrounding the textile yarn/ fiber. Depending on the curing intensity, the insulation effect of the print varies. The adhesion of the ink on the PET surface is indicated by the difference in melting peak temperature $\Delta T_{\text {washed }}$ of the photochromic prints before and after washing. Washing hence gives information about the durability of the photochromic, UV-cured surface treatment on PET.

\section{Statistical analysis}

Statistical analysis of colour measurement and DSC data was conducted in Origin 2017 from OriginLab Corporation, USA at a confidence interval of 95\%. One-way ANOVAs were used to determine the effect of washing on the crosslinking density of the ink, colour yields and rate constants of the dye. Two-way ANOVAs were used to determine the effect of the production parameters belt speed and lamp intensity, as well as the significance of their interaction.

\section{Results and discussion}

\section{Design of the UV-curable ink}

UV-Vis spectra confirm the fit of photo-initiator (absorption peak between 340 and $410 \mathrm{~nm}$ ) used in the ink formulation with the UV-LED light source (emission band of 380 to $420 \mathrm{~nm}$ ) to initiate polymer crosslinking. The normalized absorption peaks of the photochromic dye in solvent and UV-resin conform to each other. Ruby Red has an absorption peak of $500 \mathrm{~nm}$ in ethyl acetate and $490 \mathrm{~nm}$ in the UV-curable resin (Fig. S1 in $\mathrm{ESI}_{\dagger}^{\dagger}$ ). Analysis of the measured colour values, rate constants for colour development and reversion are hence made at a wavelength of $500 \mathrm{~nm}$.

\section{DSC analysis of crosslinks in the UV-curable ink}

Effect of deposited ink amount and curing settings on the crosslinking density. Thermal analysis of the interface between the printed photochromic ink and the PET fibre was measured by DSC. The cross-linked UV-ink gives no endothermic behaviour, however, it behaves as heat insulation layer, which affects the heat 
flow into PET during DSC measurement. The DSC measurement therefore gives indirect information on the polymer crosslinking density of the photochromic ink by measuring the melting temperature $\left(T_{\mathrm{m}}\right)$ of the PET fabric with UV-cured photochromic prints. For prints with increased ink deposition, or cured with higher intensity, i.e. higher lamp intensity and/or lower belt speed, the ink has a higher crosslinking density. Hence, it forms a thicker or more distinct insulation layer on the PET fabric surface. This in return increases the $T_{\mathrm{m}}$ and differences in the melting peak temperature, i.e. $\Delta T_{\mathrm{m}}$ of the PET fabric with photochromic prints on its surface compared to the untreated PET fabric. Inkjet-printed samples with different printing passes cured at a belt speed of $50 \mathrm{~mm} \mathrm{~s}^{-1}$ and $80 \%$ of the maximum lamp power (Fig. 3), showed a linear increase of $T_{\mathrm{m}}$ from $252.8^{\circ} \mathrm{C}$ for 1 pass to $255.4^{\circ} \mathrm{C}$ for ten passes. Prints with more amount of cross-linked polymer deviate more from the $T_{\mathrm{m}}$ of $252.9^{\circ} \mathrm{C}$ of untreated PET fabric as they provide progressively more insulation. Furthermore, the prints showed a more significant shift of $T_{\mathrm{m}}$ with higher curing intensity (in combination with lamp intensity and belt speed). As shown in Fig. 4 , an increase of lamp intensity, $1 \%, 25 \%$ to $80 \%$, increases the $T_{\mathrm{m}}$ of the photochromic prints on PET. The prints produced at $300 \mathrm{~mm} \mathrm{~s}^{-1}$ showed a progressive increment of $T_{\mathrm{m}}$ with $254.4^{\circ} \mathrm{C}$, $255.0{ }^{\circ} \mathrm{C}$ and $255.4{ }^{\circ} \mathrm{C}$, i.e. $\Delta T_{\mathrm{m}}$ of $1.5{ }^{\circ} \mathrm{C}, 2.1^{\circ} \mathrm{C}$ and $2.5^{\circ} \mathrm{C}$. A similarly increasing trend of $T_{\mathrm{m}}$ was detected for prints produced at $50 \mathrm{~mm} \mathrm{~s}^{-1}$ with various curing intensities but with less significant variation in $\Delta T_{\mathrm{m}}$, i.e. $2.3^{\circ} \mathrm{C}, 2.4{ }^{\circ} \mathrm{C}$ and $2.5^{\circ} \mathrm{C}$ respectively. The saturation of $T_{\mathrm{m}}$ in prints produced at $50 \mathrm{~mm} \mathrm{~s}^{-1}$ indicates that the increase of curing power cannot further increase the crosslinking density in the UV-curable ink. The inkjet-printed photochromic material accounts for $c a .10 \mathrm{wt} \%$ of the measured DSC sample. To ensure representative measurements, the $T_{\mathrm{m}}$ and the standard deviation were taken from three independently

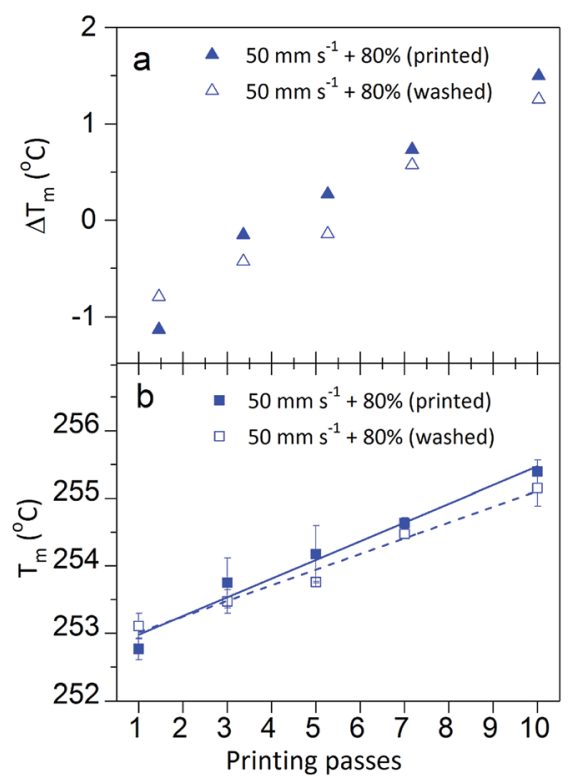

Fig. 3 (a) Melting peak temperature difference $\Delta T_{m}$ between UVcured prints and non-printed PET, after printing $(\boldsymbol{\Delta})$ and washed $(\Delta)$. The prints are cured at $50 \mathrm{~mm} \mathrm{~s}^{-1}$ belt speed and $80 \%$ lamp intensity (b) linear increase of $T_{\mathrm{m}}$ for 1 - to 10 -pass prints cured at $50 \mathrm{~mm} \mathrm{~s}^{-1}$ belt speed and $80 \%$ lamp intensity printed ( $\square$ ) and washed ( $\sqsubset$ ).

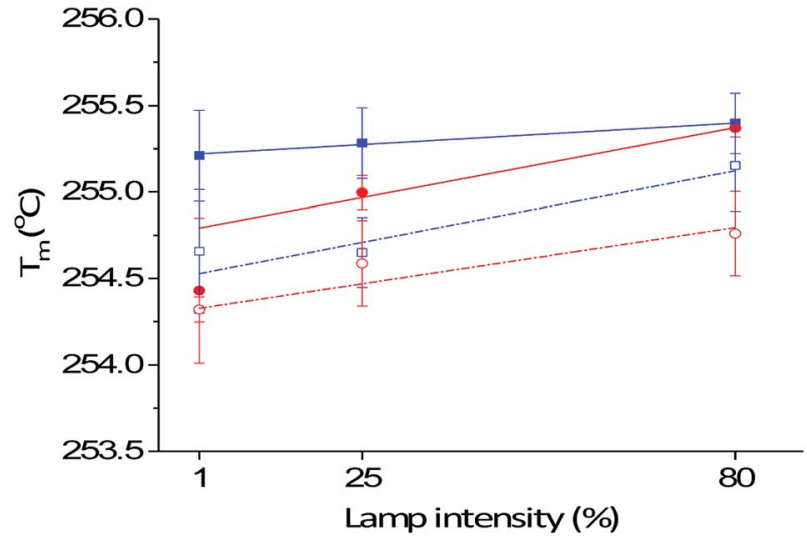

Fig. 4 Comparison of melting peak temperatures $T_{m}$ for 10-pass prints of printed samples transported at belt speed of $50 \mathrm{~mm} \mathrm{~s}^{-1}(\square)$ and $300 \mathrm{~mm} \mathrm{~s}^{-1}$ (๑) and washed samples transported at $50 \mathrm{~mm} \mathrm{~s}^{-1}$ ( $\square$ ) and $300 \mathrm{~mm} \mathrm{~s}^{-1}$ (o). Trend lines indicate the linear fit of $T_{\mathrm{m}}$ as function of lamp intensity.

measured samples. The largest standard deviation in DSC measurements is $\pm 0.5^{\circ} \mathrm{C}$, which is in general smaller than presented in Table 1. Furthermore, an indium sample was measured three times with a $T_{\mathrm{m}}$ of $156.9 \pm 0.05{ }^{\circ} \mathrm{C}$, which is comparable with literature, where indium is reported with a $T_{\mathrm{m}}$ of $156.6^{\circ} \mathrm{C}$. Moreover, in order to exclude the effect of UV-radiation on $T_{\mathrm{m}}$ of the PET fabric, the $T_{\mathrm{m}}$ of UV-cured PET fabric only, i.e. non-printed, was measured by DSC with $254.0 \pm 0.1{ }^{\circ} \mathrm{C}$. In this case, the detected variation of $\Delta T_{\mathrm{m}}\left(T_{\mathrm{m}}\right.$ of measured samples subtracted by $254.0^{\circ} \mathrm{C}$, Fig. 3(a)) which is reduced to -1 to $1.5{ }^{\circ} \mathrm{C}$, however, is still more significant than the measurement error. It can be concluded that the detected variation of $\Delta T_{\mathrm{m}}$ is small in DSC in general, but as it shows systematic variation and higher significance than the measurement error, it supports the validity of the experimental setup.

Moreover, fitting and extrapolation of the data (Fig. S2 in ESI $\dagger$ ) suggested that the $T_{\mathrm{m}}$ increased exponentially as a function of printing passes, which saturates at around 50 printed passes and a $T_{\mathrm{m}}$ of $257.1{ }^{\circ} \mathrm{C}$. This indicates that measuring $T_{\mathrm{m}}$ with DSC can approximate the amount of cross-linked UV-ink at a certain curing condition on the PET fabric.

\section{Colour performance of the photochromic textile}

Extended kinetic model for the colouration reaction. Colour measurements with the LCAM Photochrom 3 spectrophotometer have shown that the first-order kinetic model for the colouration reaction (eqn (2)) is only valid for photochromic prints with a high polymer crosslinking density, i.e. a belt speed of $50 \mathrm{~mm} \mathrm{~s}^{-1}$ and $80 \%$ lamp dosage (Fig. S3 in ESI $\dagger$ ). For cured prints with a low polymer crosslinking density, hence lower $T_{\mathrm{m}}$, polymer crosslinking continues during colour measurements and a simultaneous secondary decay mechanism occurs. The trend of deviation from normal photochromic colouration behaviour is supported by DSC measurements. The lower the speed of the transportation belt and the higher the lamp intensity for curing, the higher is the $T_{\mathrm{m}}$ and hence the insulation effect of the cured ink on PET (Fig. 4). When using 
Table 1 Melting peak temperatures $T_{\mathrm{m}}$ of 10 -pass prints at printed and washed condition for all curing setting combinations. $\Delta T_{\mathrm{m}}$ denotes the difference in $T_{m}$ between the printed and non-printed samples. $\Delta T_{\text {washed }}$ denotes the difference in $T_{m}$ between the printed and washed samples and is a measure of print durability as a result of ink-fibre adhesion

\begin{tabular}{|c|c|c|c|c|c|}
\hline Belt speed $\left(\mathrm{mm} \mathrm{s}^{-1}\right)$ & $\begin{array}{l}\text { Lamp } \\
\text { intensity (\%) }\end{array}$ & $\begin{array}{l}T_{\mathrm{m}}\left({ }^{\circ} \mathrm{C}\right) \\
\text { printed }\end{array}$ & $\begin{array}{l}T_{\text {washed }}\left({ }^{\circ} \mathrm{C}\right) \\
\text { washed }\end{array}$ & $\begin{array}{l}\Delta T_{\mathrm{m}}\left({ }^{\circ} \mathrm{C}\right) \\
T_{\mathrm{m}}-\text { UV-treated PET }\end{array}$ & $\begin{array}{l}\Delta T_{\text {washed }} \\
\left({ }^{\circ} \mathrm{C}\right)\end{array}$ \\
\hline Untreated PET & & $252.9 \pm 0.2$ & - & & \\
\hline & 25 & $255.0 \pm 0.1$ & $254.6 \pm 0.3$ & 1.0 & 0.4 \\
\hline & 80 & $255.4 \pm 0.1$ & $254.8 \pm 0.2$ & 1.4 & 0.6 \\
\hline 50 & 1 & $255.2 \pm 0.3$ & $254.7 \pm 0.4$ & 1.2 & 0.5 \\
\hline
\end{tabular}

a conveyer belt speed of $50 \mathrm{~mm} \mathrm{~s}^{-1}$, the differences in polymer crosslinking density through curing at different radiation intensities, 1,25 or $80 \%$, become less crucial. At high belt speed, i.e. $300 \mathrm{~mm} \mathrm{~s}^{-1}$, increasing lamp power plays a decisive role in the crosslinking density that is achieved. Deviation from eqn (2) for the colouration reaction is in line with the degree of polymer crosslinking in the following order, starting from the curing settings with the highest deviation:

$300 \mathrm{~mm} \mathrm{~s}^{-1} / 1 \%>300 \mathrm{~mm} \mathrm{~s}^{-1} / 25 \%>50 \mathrm{~mm} \mathrm{~s}^{-1} / 1 \%>50 \mathrm{~mm}$ $\mathrm{s}^{-1} / 25 \%>300 \mathrm{~mm} \mathrm{~s}^{-1} / 80 \%>50 \mathrm{~mm} \mathrm{~s}^{-1} / 80 \%$

The combination of highest belt speed and lowest lamp intensity, $300 \mathrm{~mm} \mathrm{~s}^{-1}$ and $1 \%$, has the highest deviation from the kinetic model in the first colouration cycle as a result of lowest polymer crosslinking density. This conforms to the colour data, which shows that UV-light exposure during colour measurement of samples with low crosslinking density decreased $\Delta K / S_{\text {colouration }}$ in the subsequent colouration cycles 2 , 3, 4 and 5 (Fig. 5). This can be a result of continuous curing and simultaneous dye degradation. High UV-radiation improves the cure speed of the resin ${ }^{32,33}$ and longer irradiance increases the degree of polymer crosslinking. ${ }^{21}$ Meanwhile, photodegradation of photochromic dyes is commonly observed under prolonged UV-irradiance. ${ }^{34-36}$

Therefore, for prints with lower polymer crosslinking density, we introduce a model that defines the kinetics of a decay mechanism as a result of continuous curing while colour measurement and reconstructs the actual colouration curve (Fig. 5). For ink systems, where UV-light is used to cure the photochromic prints with the aim to optimize curing conditions, the following extended kinetic model is proposed.

$$
\begin{aligned}
\Delta\left(\frac{K}{S}\right)_{\text {colouration }}= & {\left[\left(\frac{K}{S}\right)_{0 \text { col }}-\left(\frac{K}{S}\right)_{\infty \text { col }}\right] \mathrm{e}^{-k_{\text {colouration }} t}+\left(\frac{K}{S}\right)_{\infty \text { col }} } \\
& -\left[\left(\frac{K}{S}\right)_{0 \text { dec }}-\left(\frac{K}{S}\right)_{\infty \text { dec }}\right] \mathrm{e}^{-k_{\text {decay }} t}+\left(\frac{K}{S}\right)_{\infty \text { dec }}
\end{aligned}
$$

where $K / S_{\text {ocol }}$ is the initial colour value for the colouration reaction, $K / S_{\infty \text { col }}$ is the maximum colouration value, $K / S_{\text {odec }}$ is the initial value of the decay and $K / S_{\infty \mathrm{dec}}$ is the final value of the degrading reaction for each activation cycle. Kinetic rate constants for the colouration reaction and decay are defined as $k_{\text {colouration }}$ and $k_{\text {decay }}$, respectively.
Under the boundary condition that the processes of colouration and decay start simultaneously and at $\Delta K / S=0$, which means that $K / S_{0 \mathrm{col}}=0$ and $K / S_{\text {0dec }}=0$, eqn (3) reduces to

$$
\begin{aligned}
\Delta\left(\frac{K}{S}\right)_{\text {colouration }}= & \left(\frac{K}{S}\right)_{\infty \text { col }}\left(1-\mathrm{e}^{-k_{\text {colouration }} t}\right)-\left(\frac{K}{S}\right)_{\infty \text { dec }} \\
& \left(1-\mathrm{e}^{-k_{\text {decay }} t}\right)
\end{aligned}
$$

Via continuous UV-exposure over $1500 \mathrm{~s}$ it could be excluded that the decolouration reaction interferes with the observed decay mechanism during the colouration reaction (Fig. S5 in ESI $\dagger$ ). Rate constants of the decay mechanism $k_{\text {decay }}$ were fitted as exponential decay during UV-exposure, i.e. to the decrease in

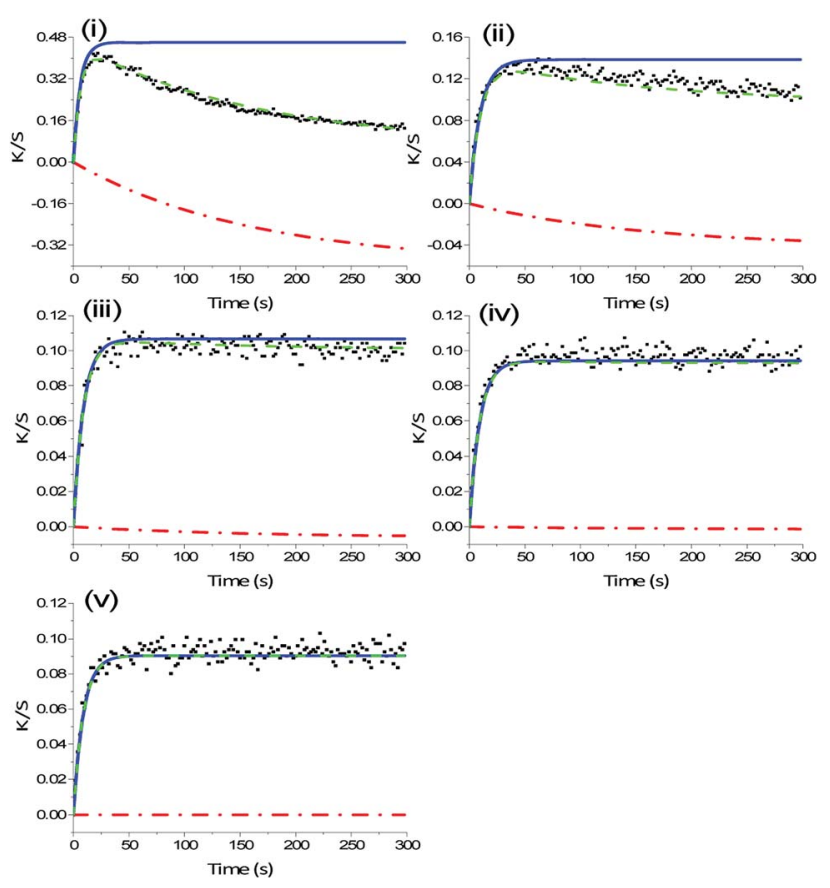

Fig. 5 Extended model for reconstruction of photochromic colouration throughout colouration cycles 1-5 $(\mathrm{i}-\mathrm{v})$ of a print with $19 \mathrm{~g}$ $\mathrm{m}^{-2}$ deposited ink cured at belt speed of $300 \mathrm{~mm} \mathrm{~s}^{-1}$ and $1 \%$ lamp intensity. Measured $K / S$ data ( $(\mathbf{)})$ consists of reaction processes of decay $(-)$ and colouration $(-)$ during colour measurement. The calculated sum of decay and colouration ( --$)$ validates the model. For this print degradation becomes statistically ineffective in activation cycle 5 (v). 
$K / S_{\text {colouration }}$ throughout the five UV-exposure cycles during the colouration reaction, which add up to $1500 \mathrm{~s}$.

The extended kinetic model, eqn (4), has been applied to the colour data of inkjet-printed samples with a deviation from eqn (2), i.e. $\mathrm{R}^{2}<0.95$, to reconstruct the $K / S_{\text {colouration values and }}$ $k_{\text {colouration, as well as to determine the extent of decay depending }}$ on the curing setting and applied amount of ink. An increasing amount of ink influences the secondary decay of the dye throughout colour measurement. It is a common trend for all curing settings that the lower the amount of applied ink, the higher is $k_{\text {decay. }}$. Prints cured at a belt speed of $50 \mathrm{~mm} \mathrm{~s}^{-1}$ and $1 \%$ lamp power show decreasing $k_{\text {decay }}$ from $0.017 \mathrm{~s}^{-1}$ to 0.014 $\mathrm{s}^{-1}$ to $0.004 \mathrm{~s}^{-1}$ for 3,5 and 10 printing passes, respectively. With increasing layer thickness oxygen diffusion is aggravated and the primarily affected surface area is proportionally reduced. As presented in Table 2, the secondary decay decreases with stronger curing conditions from $k_{\text {decay }}=0.006 \mathrm{~s}^{-1}$ for $300 \mathrm{~mm} \mathrm{~s}^{-1}$ and $1 \%$ to $0.002 \mathrm{~s}^{-1}$ for $50 \mathrm{~mm} \mathrm{~s}^{-1}$ and $25 \%$. Also, variation in $k_{\text {decay }}$ is higher when transported at $300 \mathrm{~mm} \mathrm{~s}^{-1}$. Hence, the decreasing trend of variation in $k_{\text {decay }}$ and rates with increasing crosslinking density of prints directly correlates with the trend of deviation from eqn (2).

The intensity of the light source used for exposure in the LCAM Photochrom 3 spectrophotometer with $8 \mathrm{~mW} \mathrm{~cm}^{-2}$ at sample position is 750-times lower than the UV-LED lamp intensity, i.e. approx. 10-times lower than 1\% intensity of the curing lamp. Also, exposure of the prints to UV-light stretches over $300 \mathrm{~s}$ during colour measurement instead of approx. $1 \mathrm{~s}$ during the curing process. Both low irradiation intensity and long exposure time of the photochromic dye in a liquid resin cause rapid degradation as a result of photo-oxidation. A partially liquid resin has a lower viscosity, which facilitates oxygen diffusion. ${ }^{33}$ It can be assumed that dye degradation as a result of photo-oxidation occurs in the soft component of the resin, which is more prominent compared to when the dye is fixed in a solid matrix. However, cut-off effects of the dyecarrying medium, i.e. shift in reflectance properties as a result of changed degree of cure and media thickness have also been investigated. ${ }^{\mathbf{1 0 , 3 7}}$ We therefore consider the observed decrease a secondary decay, which obviously reduces $\Delta K / S$ and colour reaction kinetics.

Effect of amount of ink deposition on the colouration reaction. It was found that the achieved colour intensity $\Delta K /$ $S_{\text {colouration }}$ of printed and washed samples was linear towards the deposited amount of photochromic ink irrespective of curing conditions (Fig. 7), which agrees with the linearly increasing $T_{\mathrm{m}}$ as function of ink amount. Stronger colour yields with increasing ink amount are visible by eye for both printed and washed samples as can be seen in Fig. 1(c). According to Viková et al. $^{13}$ and Periyasamy et al., ${ }^{38}$ deeper shades are expected with increased layer thickness as a result of lowered surface-bulk ratio and hence, less colourless reflection. Aldib ${ }^{15}$ also observed the trend of a continuous increase in colour yield from 1 to 10 inkjet printing passes of the same dye type but for solvent-based ink. This indicates that the crosslinking density and the adhesion of the UV-resin to the textile surface are not affected by the amount of ink deposition.

The amount of printed photochromic ink on PET influences the kinetics of the colouration reaction. Similar to the trend in decrease in $k_{\text {decay }}$, an increasing amount of applied material lowers $k_{\text {colouration }}$ as seen in Fig. 6(b) for prints cured at a belt speed of $50 \mathrm{~mm} \mathrm{~s}^{-1}$ and $80 \%$ of lamp intensity. A decrease in $k_{\text {colouration }}$ as function of printing passes can be explained by weakened activation of the photochromic dye with increased layer thickness. ${ }^{10}$ Washing has a significant effect on the colouration behaviour of the photochromic prints. Both $\Delta K /$ $S_{\text {colouration }}$ and $k_{\text {colouration }}$ significantly decrease as a result of a washing cycle. The reduced colour yield $\Delta K / S_{\text {colouration }}$ approximates $40 \%$ of the initial value for all printing passes from 1 to 10 . With a decrease of approx. $20 \%$ at 10 passes

Table 2 Colour yields $\Delta K / S_{\text {colouration }}$ and rate constants $k_{\text {colouration, }} k_{\text {decolouration }}$ and $k_{\text {decay }}$ of 10 -pass prints for all curing settings for printed and washed samples in activation cycle 1 . Remained $\Delta K / S$ presents the percentage-wise residual colour yield $\Delta K / S_{\text {colouration }}$ after a washing process

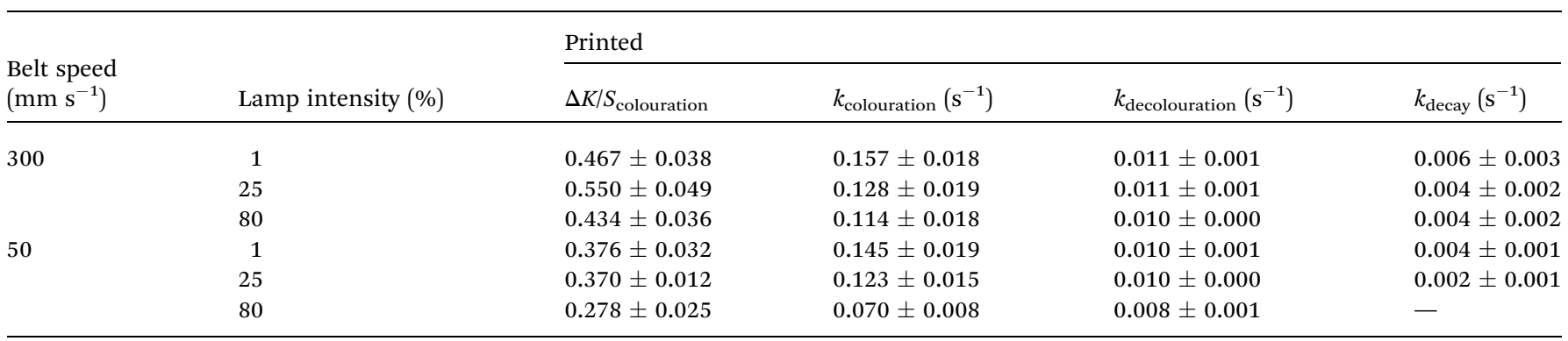

\begin{tabular}{|c|c|c|c|c|c|}
\hline $\begin{array}{l}\text { Belt speed } \\
\left(\mathrm{mm} \mathrm{s}^{-1}\right)\end{array}$ & Lamp intensity (\%) & \multicolumn{3}{|l|}{ Washed } & Remained $\Delta K / S(\%)$ \\
\hline & 25 & $0.266 \pm 0.007$ & $0.086 \pm 0.014$ & $0.010 \pm 0.001$ & 48.3 \\
\hline & 80 & $0.213 \pm 0.020$ & $0.066 \pm 0.013$ & $0.009 \pm 0.001$ & 49.0 \\
\hline 50 & 1 & $0.206 \pm 0.034$ & $0.077 \pm 0.020$ & $0.009 \pm 0.001$ & 54.8 \\
\hline
\end{tabular}


compared to $40 \%$ at 1 pass, $k_{\text {colouration }}$ is less affected with increasing layer thickness.

Effect of crosslinking density on the colouration reaction. The production parameters belt speed and lamp intensity have a significant effect on $\Delta K / S_{\text {colouration }}$ and rate constants $k_{\text {colouration }}$ and $k_{\text {decay }}$. In Fig. 7, curing parameters belt speed and lamp intensity, $300 \mathrm{~mm} \mathrm{~s}^{-1}+1 \%, 50 \mathrm{~mm} \mathrm{~s}^{-1}+1 \%$ and $50 \mathrm{~mm}$ $\mathrm{s}^{-1}+80 \%$ represent settings, which result in low, intermediate and high crosslinking density, respectively. Prints with high crosslinking density have experienced strongest curing, which increases the rigidity of the UV-resin but may also cause dye degradation. As a result, $\Delta K / S_{\text {colouration }}$ is lowered. As seen in Table 2 , highest $\Delta K / S_{\text {colouration }}$ are achieved with curing at belt speed of $300 \mathrm{~mm} \mathrm{~s}^{-1}$ and low lamp intensity, i.e. $K / S_{\text {colouration }}=$ 0.47 with $1 \%$ and $K / S_{\text {colouration }}=0.55$ with $25 \%$.

A high crosslinking density provides good ink-substrate adhesion, which is seen in high remaining $\Delta K / S_{\text {colouration }}$ values after washing. The remaining $\Delta K / S$ of 10 -pass prints after a washing process is highest for strongest curing conditions, i.e. $55.5 \%$ for $50 \mathrm{~mm} \mathrm{~s}^{-1}$ and $80 \%$. Washing decreases $\Delta K /$ $S_{\text {colouration }}$ significantly for all curing conditions, with maximum remaining $55.5 \%$ to minimum $48.3 \%$ for 10 -pass prints. It can be concluded that stronger curing conditions result in low absolute $\Delta K / S_{\text {colouration }}$ and low $k_{\text {colouration }}$ and $k_{\text {decolouration. A }}$ low polymer crosslinking density of the carrier matrix exhibits a higher amount of soft component in the photopolymer, which enables more flexibility and free volume for faster switching of the photochromic dye molecule. ${ }^{24,27}$ A comparison of colour intensities and colouration rates between the six combinations of curing settings reveals that the setting with the lowest curing intensity, i.e. belt speed of $300 \mathrm{~mm} \mathrm{~s}^{-1}$ and lamp intensity of $1 \%$, is the most favourable. High $\Delta K / S$ and fast kinetic switching with $k_{\text {colouration }}$ of $0.157 \mathrm{~s}^{-1}$ after printing and $0.109 \mathrm{~s}^{-1}$ after
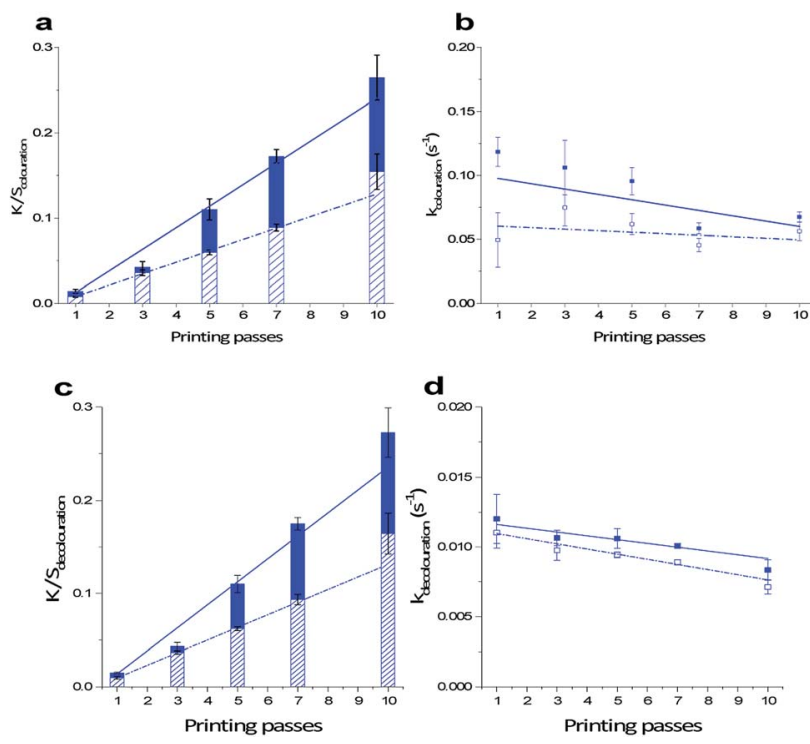

Fig. 6 Trend lines indicate (a) linear increase of $\Delta K / S_{\text {colouration, (b) }}$ successive decrease in $k_{\text {colouration, }}$ (c) linear increase of $\Delta K / S_{\text {decolouration }}$ and (d) successive decrease in $k_{\text {decolouration }}$ as function of printing passes from 1 to 10 cured at a belt speed of $50 \mathrm{~mm} \mathrm{~s}^{-1}$ and $80 \%$ of lamp intensity printed $(\boldsymbol{\square})$ and washed $(\square)$.

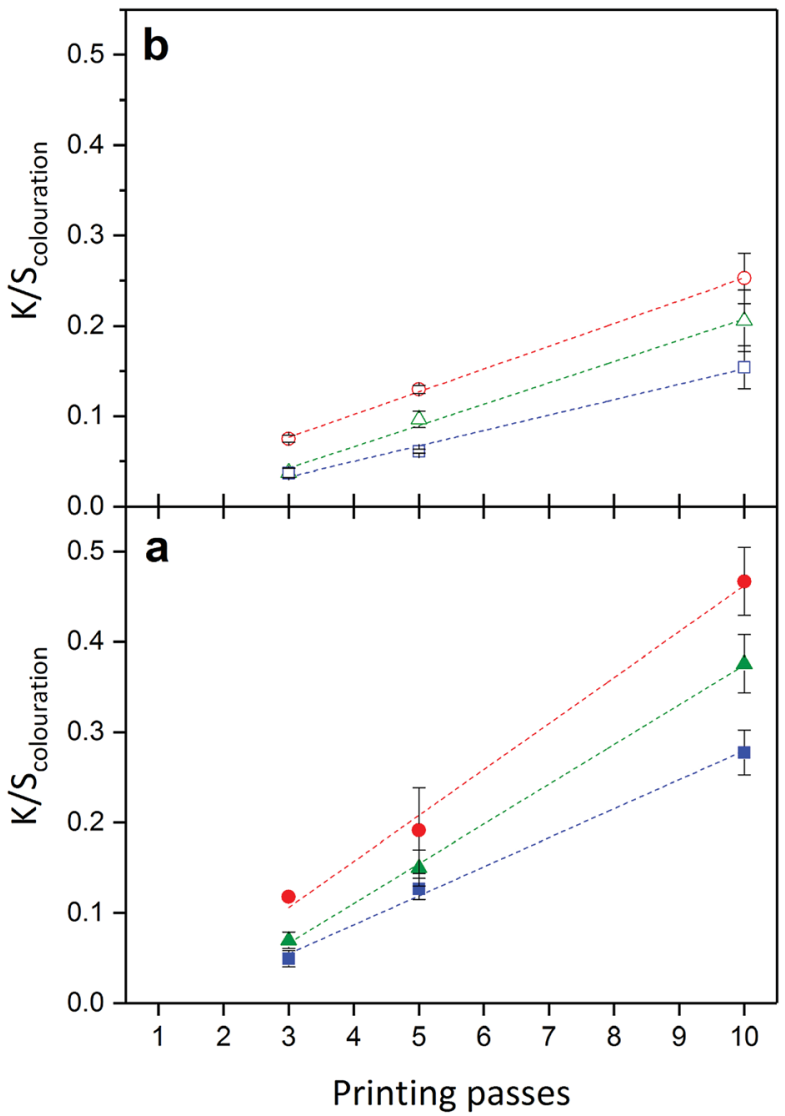

Fig. 7 Linear increase of $\Delta K / S_{\text {colouration }}$ as a function of printing passes for low, intermediate and strong curing conditions, i.e. $300 \mathrm{~mm} \mathrm{~s}^{-1}+$ $1 \%(\bullet, 0), 50 \mathrm{~mm} \mathrm{~s}^{-1}+1 \%(\Delta, \Delta)$ and $50 \mathrm{~mm} \mathrm{~s}^{-1}+80 \%(\bullet,-$,$) , respectively$ after printing (a) and washing (b).

washing between the uncoloured and coloured state of the textile UV-sensor is achieved. A higher cross-linked resin results in lowered rates of colouration with i.e. $k_{\text {colouration }}$ of $0.070 \mathrm{~s}^{-1}$ before and $0.047 \mathrm{~s}^{-1}$ after washing for 10-pass prints cured at $50 \mathrm{~mm} \mathrm{~s}^{-1}$ and $80 \%$.

Viková $^{\mathbf{1 0}}$ applied naphthopyran dyes on textiles in a screenprinting paste and achieved colouration rates of $0.09 \mathrm{~s}^{-1}$ and $0.25 \mathrm{~s}^{-1}$ with a concentration of $0.25 \mathrm{wt} \%$ at lamp intensity of $800 \mu \mathrm{W} \mathrm{cm}^{-2}$. The same dyes applied with a concentration of $1 \mathrm{wt} \%$ in a spin dope, which was melt-blown to a non-woven achieved colouration rates between $0.01 \mathrm{~s}^{-1}$ and $0.03 \mathrm{~s}^{-1} .^{10}$ With respect to different concentrations and activation powers it can be assumed that similar dye types to Reversacol Ruby Red applied via a UV-cured matrix obtain $k_{\text {colouration, which are }}$ higher than in a fibre structure but lower than in a water-based and thermally cured acrylate paste.

Effect of amount of ink deposition on the decolouration reaction. The decolouration reaction as shown in Fig. 2 of naphthopyran dye Ruby Red is a thermal reaction, which takes place in the dark and therewith is independent from the colouration reaction. ${ }^{27,39}$ Decolouration yields $\Delta K / S_{\text {decolouration, }}$ which correspond to $\Delta K / S_{\text {colouration, and independent decolou- }}$ ration rates $k_{\text {decolouration, }}$ are calculated using eqn (2). A significant difference is seen for $\Delta K / S_{\text {decolouration }}$ after printing and 
after a washing cycle. The same behaviour as for $\Delta K / S_{\text {colouration }}$ is seen for decolouration intensities. As seen in Fig. 6(c), $\Delta K /$ $S_{\text {decolouration }}$ increase linearly as function of printing passes and exhibit approx. $60 \%$ of the initial yields after washing. It is noted that for small amounts of printed ink, i.e. 1 and 3 printing passes, only $35 \%$ and $15 \%$ of the initial $\Delta K / S_{\text {decolouration }}$ is lost during the washing process, respectively.

With an increasing amount of printed ink, $k_{\text {decolouration }}$ to revert the photochromic dye back to its uncoloured state are successively lowered by $c a$. 30\% when cured with low speed and high lamp intensity, i.e. $50 \mathrm{~mm} \mathrm{~s}^{-1}$ and $80 \%$. $k_{\text {decolouration }}$ decreases from $0.012 \mathrm{~s}^{-1}$ for 1-pass prints to $0.008 \mathrm{~s}^{-1}$ for 10 pass prints. A washing process has a significant effect and lowers the decolouration rates by $10-15 \%$ for all printing passes (Fig. 6(d)). Same as for $k_{\text {colouration, }} k_{\text {decolouration }}$ is influenced by the thickness of the applied ink on the textile surface. An increase in layer thickness decreases the reaction rate.

Effect of crosslinking density on the decolouration reaction. The crosslinking density has a statistically significant effect on $\Delta K / S_{\text {decolouration }}$ irrespective of the amount of printed ink, i.e. 1 to 10 passes, on PET before and after washing. The higher the speed of the conveyer belt, the higher is $\Delta K / S_{\text {decolouration. It is }}$ also valid, that the lower the UV-dosage during curing, the higher is the expected $\Delta K / S$. Decolouration rates are most affected by strong curing conditions, that means $50 \mathrm{~mm} \mathrm{~s}^{-1}$ belt speed and $80 \%$ lamp intensity. $k_{\text {decolouration follows the same }}$

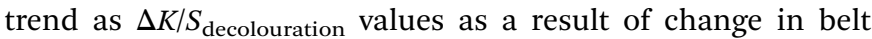
speed and lamp power, i.e. crosslinking density. The lower the crosslinking density, the least rigid is the UV-resin and hence the higher is $k_{\text {decolouration }}$ of the photochromic dye to its uncoloured state. For 10-pass prints transported with a belt speed of $300 \mathrm{~mm} \mathrm{~s}^{-1}$ and cured with a lamp intensity of $1 \%$ $k_{\text {decolouration }}$ is nearly unchanged with a rate of $0.011 \mathrm{~s}^{-1}$ after printing and $0.010 \mathrm{~s}^{-1}$ after washing as listed in Table 2. Curing at this condition allows the production of photochromic prints with an expected $\Delta K / S=0.25$ after washing and switching on of the photochromic effect at a rate of $0.11 \mathrm{~s}^{-1}$. Impact on the change in $k_{\text {colouration }}$ and $k_{\text {decolouration }}$ achieved by differences in conveyer belt speeds and lamp intensities during the curing process is less compared to other means of polymer architecture. Ercole et $a ._{.}{ }^{25}$ and Malic et $a .^{26}$ studied that mid placement of the photochromic dye in homo-polymers can optimize photochromic kinetics. A similar effect can be achieved by tuning the polymer length and rigidity of the polymer matrix. Whereas, via polymer conjugation and, of course with respect to the generic switching speed of the chosen dye molecule, decolouration rates of a naphthopyran dye can be doubled to increased up to a five-fold from 0.01 to $0.05 \mathrm{~s}^{-1},{ }^{26}$ by variation of fabrication parameters during curing approx. 1.4-times faster $k_{\text {decolouration }}$ can be obtained.

\section{Durability and adhesion of the photochromic ink}

Effect of deposited ink amount and curing settings on the print durability. Washing, which is an essential feature in textile applications, gives information on the durability and adhesion of a functional treatment on the textile surface. Here, the difference in $T_{\mathrm{m}}$ of the photochromic prints can be applied as a measure of durability in terms of adhesion of the ink on the PET surface. The durability of photochromic prints is assessed by measurement of $T_{\mathrm{m}}$ in DSC after a standard washing cycle (ISO 6330), i.e. the difference between $T_{\mathrm{m}}$ and the melting peak temperature after washing $\left(T_{\text {washed }}\right)$, i.e. $\Delta T_{\text {washed }}$. The more cross-linked UV-ink is left on the PET surface after a washing cycle, the more durable is the functional surface, and hence, the smaller the $\Delta T_{\text {washed. }}$. The effect of washing ( $\left.\Delta T_{\text {washed }}\right)$ gives indirect information on the durability of the cross-linked photochromic ink and its adhesion to the surface of PET.

Washed samples exhibit a statistically significantly lowered melting temperature $T_{\text {washed }}$ regardless of the curing condition, as shown in Fig. 4 . The decreased $T_{\text {washed, }}$, as listed in Table 1, is a measure for durability in terms of adhesion of the cured photochromic print to the PET fabric surface. While the $\Delta T_{\text {washed }}$ for prints cured at $300 \mathrm{~mm} \mathrm{~s}^{-1}$ with $1 \%$ lamp power remains nearly unchanged with a decrease of $0.1^{\circ} \mathrm{C}$, for prints transported at a belt speed of $50 \mathrm{~mm} \mathrm{~s}^{-1}$, the trend of the $T_{\mathrm{m}}$ as a function of lamp intensity changes after washing. Photochromic prints that underwent strongest curing at $80 \%$ of the maximum lamp power had almost no change in $\Delta T_{\text {washed }}\left(0.2^{\circ} \mathrm{C}\right.$ within the measurement error). Prints that have been cured at both $25 \%$ and $1 \%$ lamp intensity have a $T_{\text {washed }}$ of $254.7^{\circ} \mathrm{C}$. This gives a $\Delta T_{\text {washed }}$ of $0.6{ }^{\circ} \mathrm{C}$ and $0.5{ }^{\circ} \mathrm{C}$ for lamp powers $25 \%$ and $1 \%$, respectively. The result for the samples processed with $50 \mathrm{~mm} \mathrm{~s}^{-1}$ suggested that higher lamp power could increase the crosslinking density in the printed UV-ink.

Furthermore, the combination of $T_{\text {washed }}$ and $\Delta T_{\text {washed }}$ assesses the durability of the functional layer with different crosslinking densities, e.g., the combination of high $T_{\text {washed }}$ and small $\Delta T_{\text {washed }}$ gives an indication for a durable photochromic layer with high crosslinking density in the prints. Prints cured at $50 \mathrm{~mm} \mathrm{~s}^{-1}$ and $80 \%$ have the highest $T_{\text {washed }}$ of $255.2{ }^{\circ} \mathrm{C}$ and a $\Delta T_{\text {washed }}$ of $0.2{ }^{\circ} \mathrm{C}$. It implies that a higher polymer crosslinking density is achieved as the print creates a strong insulation layer on the PET surface. Prints cured with the lowest curing intensity, i.e. $300 \mathrm{~mm} \mathrm{~s}^{-1}$ and $1 \%$, exhibit a lower polymer crosslinking density, but are durable and flexible as the $T_{\text {washed }}$ is the lowest with $254.3{ }^{\circ} \mathrm{C}$ (with $\Delta T_{\text {washed }}$ of $0.1{ }^{\circ} \mathrm{C}$ ).

As shown in Fig. 3(b), washing has an effect on the inkjetprinted samples cured at a belt speed of $50 \mathrm{~mm} \mathrm{~s}^{-1}$ and $80 \%$ lamp intensity from 1 to 10 printing passes. The slope of the linear fit of $T_{\text {washed }}$ as a function of printing passes is slightly lower than the fit of $T_{\mathrm{m}}$. However, changes in $T_{\text {washed }}$ from $253.8{ }^{\circ} \mathrm{C}$ to $253.5{ }^{\circ} \mathrm{C}\left(\Delta T_{\text {washed }}\right.$ of $\left.0.3{ }^{\circ} \mathrm{C}\right)$ for 3 passes and from $255.4{ }^{\circ} \mathrm{C}$ to $255.2^{\circ} \mathrm{C}\left(\Delta T_{\text {washed }}\right.$ of $\left.0.2^{\circ} \mathrm{C}\right)$ for 10 passes are small (Fig. 3(b)).

The durability of the functional layer $\left(\Delta T_{\text {washed }}\right)$ related to process parameters can be confirmed by the difference in $\Delta K / S$ from colour measurements after washing. The samples produced at $300 \mathrm{~mm} \mathrm{~s}^{-1}$ and $1 \%$ exhibit $54.1 \%$ of the initial colour yield, whereas the sample produced at $50 \mathrm{~mm} \mathrm{~s}^{-1}$ and $80 \%$ preserves $55.5 \%$ of its initial colour after one wash. Both curing conditions show decent durability assuming that the colour performance is in linear relationship with the amount of UV-ink printed (remained) on the PET fabric surface. 
Furthermore, the weight of printed and washed functional layers is complimentary to DSC and colour measurements (ESI $\dagger$ ). As shown in Fig. S5, $\dagger$ the samples produced with various amounts of ink deposition cured at $50 \mathrm{~mm} \mathrm{~s}^{-1}$ and $80 \%$ were weighed after printing and washing. The weight of the functional layer, with the exception of 1-pass prints (measured weight loss of $100 \%$ ), decreased by an average of $23 \pm 12 \%$. The weight decrease is due to a loss of the photochromic print during washing, which correlates with the decreasing trends in $T_{\mathrm{m}}$ and $\Delta K / S$.

The result confirms that a belt speed of $50 \mathrm{~mm} \mathrm{~s}^{-1}$ and lamp intensity of $80 \%$ are valid process parameters to obtain a durable functional layer with high crosslinking density in prints. In summary, both a belt speed of $300 \mathrm{~mm} \mathrm{~s}^{-1}$ and lamp intensity of $1 \%$ and a belt speed of $50 \mathrm{~mm} \mathrm{~s}^{-1}$ and lamp intensity of $80 \%$ can produce a durable functional layer but the latter results in a higher crosslinking density in prints. The $T_{\text {washed }}$ of the photochromic prints on PET after one washing cycle reaches a mean value of $254.7{ }^{\circ} \mathrm{C}$ for all curing settings. Here, statistical analysis shows that differences in belt speed and lamp intensity are insignificant in regards to $T_{\mathrm{m}}$ and $T_{\text {washed }}$. Despite an increasing trend in crosslinking density with lower belt speeds and higher lamp powers, it can be assumed that stronger curing intensities than $300 \mathrm{~mm} \mathrm{~s}^{-1}$ and $1 \%$ are not more beneficial for the durability of the print.

\section{Conclusions}

The colour performance of a novel UV-curable and UVresponsive smart textile is optimized using the combination of two challenging functions of electromagnetic radiation, where high energy UV-rays both cure and activate UV-sensitive naphthopyran dye Ruby Red. Tuning of the crosslinking density of the photochromic ink and hence the dye kinetics using fabrication parameters is an important step towards the design of a textile UV-sensor. Irrespective of the deposited ink amount on the PET surface between 1 and 10 printing passes, curing with the highest belt speed of $300 \mathrm{~mm} \mathrm{~s}^{-1}$ combined with the lowest lamp intensity $1 \%$ of the pilot-scale inkjet printing system achieves high colour yields $\Delta K / S$ with 0.47 after printing and 0.25 after washing, fastest isomerization with

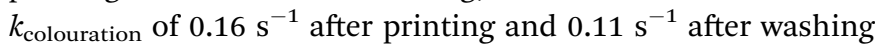
and good durability after washing with $54.1 \%$ of the initial $\Delta K /$ $S$, despite lowest polymer crosslinking density. The effect of the solid photopolymer resin compared to waterborne or solventbased carriers for a photochromic dye, similar to Reversacol Ruby Red, applied on textiles results in slower switching rates between the coloured and uncoloured states, but faster than in mass-dyed and melt-spun fabrics. Our findings add to the general trend of enhancing switching rates between the different isomers of photochromic dyes, as well as the overall colour yield as a result of reduced rigidity of the polymer matrix. Via a new kinetic model, which takes a decay mechanism of the dye upon colour measurement into account, the colour performance can be predicted and tuned based on fabrication parameters. Beyond tuning of colour kinetics, the novel and industrially applicable approach of UV-LED curing of photochromic inkjet ink is favourable in regards to the flexibility, resource-efficiency and cost-effectiveness of the production process. Inkjet printing combined with UV-LED curing has large potential to trigger innovative products and to promote the small-batch production of functional high-end and smart textiles.

\section{Conflicts of interest}

There are no conflicts to declare.

\section{Acknowledgements}

This work was supported by Borås Stad, Sparbankstifelsen Sjuhärad, TEKO (The Swedish Textile and Clothing Industries Association) and SST (Stiftelsen Svensk Textilforskning).

\section{References}

1 A. Schwarz, L. Van Langenhove, P. Guermonprez and D. Deguillemont, Text. Prog., 2010, 42, 99-180.

2 J. Wei, X. Jiao, T. Wang and D. Chen, J. Mater. Chem. C, 2015, 3, 7597-7603.

3 D. Wen, Y. Liu, C. Yue, J. Li, W. Cai, H. Liu, X. Li, F. Bai, H. Zhang and L. Lin, RSC Adv., 2017, 7, 54741-54746.

4 H. Liu, Y. Lv, S. Li, F. Yang, S. Liu, C. Wang, J.-Q. Sun, H. Meng and G.-G. Gao, J. Mater. Chem. C, 2017, 5, 93839388.

5 D. Kathiravan, B.-R. Huang and A. Saravanan, J. Mater. Chem. C, 2017, 5, 5239-5247.

6 M. Qin, Y. Huang, F. Li and Y. Song, J. Mater. Chem. C, 2015, 3, 9265-9275.

7 J. C. Crano and R. J. Guglielmetti, Organic Photochromic and Thermochromic Compounds, Springer, New York, 2006.

8 A. F. Little and R. M. Christie, Color. Technol., 2011, 127, 275281.

9 T. Feczkó, K. Samu, K. Wenzel, B. Neral and B. Voncina, Color. Technol., 2013, 129, 18-23.

10 M. Viková, PhD thesis, Heriot-Watt University, 2011.

11 M. Aldib and R. M. Christie, Color. Technol., 2013, 129, 131143.

12 M. Aldib and R. M. Christie, Color. Technol., 2011, 127, 282287.

13 M. Viková, A. P. Periyasamy, M. Vik and A. Ujhelyiová, J. Text. Inst., 2016, 1-6, DOI: 10.1080/00405000.2016.1251290.

14 H. N. Doan, H. Tsuchida, T. Iwata, K. Kinashi, W. Sakai, N. Tsutsumi and D. P. Huynh, RSC Adv., 2017, 7, 3306133067.

15 M. Aldib, Color. Technol., 2015, 131, 172-182.

16 R. Fu, J. Shi, E. Forsythe and M. Srour, Opt. Eng., 2016, 55, 124105.

17 M. M. El-Molla, Dyes Pigm., 2007, 74, 371-379.

18 O. A. Hakeim, A. A. Arafa, M. K. Zahran and L. A. W. Abdou, Colloids Surf., A, 2014, 447, 172-182.

19 M. B. V. Shukla, D. K. Singh and R. Shukla, Pigm. Resin Technol., 2004, 33, 272-279. 
20 R. Schwalm, L. Häußling, W. Reich, E. Beck, P. Enenkel and K. Menzel, Prog. Org. Coat., 1997, 32, 191-196.

21 S. Agarwal, PhD thesis, Technical University Darmstadt, 2011.

22 A. H. L. Lin, Pigm. Resin Technol., 2004, 33, 280-286.

23 H. Bouas-Laurent and H. Dürr, Pure Appl. Chem., 2001, 73, 639.

24 G. Such, R. A. Evans, L. H. Yee and T. P. Davis, J. Macromol. Sci., Polym. Rev., 2003, 43, 547-579.

25 F. Ercole, N. Malic, S. Harrisson, T. P. Davis and R. A. Evans, Macromolecules, 2010, 43, 249-261.

26 N. Malic, J. A. Campbell and R. A. Evans, Macromolecules, 2008, 41, 1206-1214.

27 K. Mutoh, Y. Kobayashi and J. Abe, Dyes Pigm., 2017, 137, 307-311.

28 F. Ercole, T. P. Davis and R. A. Evans, Macromolecules, 2009, 42, 1500-1511.

29 R. A. Evans, T. L. Hanley, M. A. Skidmore, T. P. Davis, G. K. Such, L. H. Yee, G. E. Ball and D. A. Lewis, Nat. Mater., 2005, 4, 249-253.
30 M. Vik and M. Viková, A method and device for fatigue testing of photochromic, fluorescent or phosphorescent dyes, EP3077811A1, October 2016.

31 M. Viková and M. Vik, Adv. Chem. Eng. Sci., 2011, 1, 224-230.

32 P. Glöckner, Radiation Curing: Coatings and Printing Inks; Technical Basics, Applications and Trouble Shooting, Vincentz Network, Hannover, 2008.

33 C. Decker, Macromol. Rapid Commun., 2002, 23, 1067-1093. 34 L. H. Peng, R. H. Guo, S. X. Jiang, J. W. Lan, Y. F. He and X. M. Huang, Fibers Polym., 2015, 16, 1312-1318.

35 T. Feczkó, M. Kovács and B. Voncina, J. Photochem. Photobiol., A, 2012, 247, 1-7.

36 M.-Q. Zhu, L. Zhu, J. J. Han, W. Wu, J. K. Hurst and A. D. Q. Li, J. Am. Chem. Soc., 2006, 128, 4303-4309.

37 D. Mulligan, Cure Monitoring for Composites and Adhesives, Smithers Rapra Publishing, Shrewsbury, 2003.

38 A. P. Periyasamy, M. Vikova and M. Vik, Text. Prog., 2017, 49, 53-136.

39 C. M. Sousa, J. Berthet, S. Delbaere, A. Polónia and P. J. Coelho, J. Org. Chem., 2015, 80, 12177-12181. 